\title{
Soliton dynamics in damped and forced Boussinesq equations
}

\author{
E. Arévalo, Yu. Gaididei[ [] and F. G. Mertens \\ Physikalisches Institut, Universität Bayreuth, D95440 Bayreuth, Germany
}

\begin{abstract}
We investigate the dynamics of a lattice soliton on a monatomic chain in the presence of damping and external forces. We consider Stokes and hydrodynamical damping. In the quasi-continuum limit the discrete system leads to a damped and forced Boussinesq equation. By using a multiple-scale perturbation expansion up to second order in the framework of the quasi-continuum approach we derive a general expression for the first-order velocity correction which improves previous results. We compare the soliton position and shape predicted by the theory with simulations carried out on the level of the monatomic chain system as well as on the level of the quasi-continuum limit system. For this purpose we restrict ourselves to specific examples, namely potentials with cubic and quartic anharmonicities as well as the truncated Morse potential, without taking into account external forces. For both types of damping we find a good agreement with the numerical simulations both for the soliton position and for the tail which appears at the rear of the soliton. Moreover we clarify why the quasi-continuum approximation is better in the hydrodynamical damping case than in the Stokes damping case.

PACS: 63.10.+a Lattice dynamics: General theory and 05.45.Yv Solitons
\end{abstract}

\section{Introduction}

There is a long-standing interest in the dynamical and thermodynamical properties of anharmonic monatomic and diatomic chains ( see e.g. 
[1, 2, 3, 4, 5, 6]). It was shown that these anharmonic chains can bear low-energy excitations which are solutions of a Boussinesq type equation (in the long-wavelength approximation). For realistic interatomic potentials these soliton-like excitations are supersonic and correspond to a compression of the chain where the relation between amplitude (or width) and velocity depends on the form of the interatomic potential. They are very robust and propagate without energy loss, and their collisions are almost elastic even beyond the range of validity of the continuum approximation. Due to their robust character the soliton excitations are important in the coherent energy transfer and they have been used to explain energy transport in DNA [7]. There is also a growing evidence that nonlinear excitations participate in the heat conduction of anisotropic dielectric crystals [8, 9, 10, 11]. The non-diffusive heat flow was attributed to modified Korteweg-de-Vries solitons in 11]. The role of breathers for the thermal conductivity was studied in [12].

So far the main attention was paid to soliton dynamics in the absence of dissipation. However, the dissipation influences significantly the solitons, changing their shape and velocity. An external driving force is therefore necessary to sustain a soliton in steady state. The dynamics of slowly varying solitary wave solutions of the damped Korteweg-de-Vries equation was investigated by using the methods of inverse scattering theory [15], a multiple-scale perturbation expansion [16], and a Green's function formalism [17]. The soliton motion in Toda chains in the presence of dissipation and driving forces was studied in [13, 14].

The objective of the present paper is to study properties of solitary waves in damped anharmonic lattices. We investigate two types of damping: Stokes friction (in Ref. [13] it is called outer friction) and hydrodynamical (or internal) friction [18]. We study the case of potentials with power-like anharmonicities as well as the case of a truncated Morse potential. We compare the results of numerical simulations, which are carried out for discrete anharmonic lattices as well as for the quasi-continuum Boussinesq system, with the results of a multiple-scale perturbation expansion obtained in the framework of the quasi-continuum approximation. 


\section{System and equations of motion}

We consider a chain of equally spaced particles of mass $M(M=1)$ with interatomic spacing $a(a=1)$ and displacement from equilibrium $x_{n}$. The Lagrangian of our system is given by

$$
L=T-U-U_{\text {ext }} .
$$

Here

$$
T=\frac{1}{2} \sum_{n} \dot{x}_{n}^{2}(t)
$$

is the kinetic energy $\left(\dot{x} \equiv \frac{d x}{d t}\right)$,

$$
U=\sum_{n} V\left(x_{n+1}-x_{n}\right)
$$

is the potential energy. We consider a potential between first neighbors of two types: the power-like potential

$$
\begin{aligned}
V(r) & =V_{\text {harm }}+V_{\text {anh }}, \\
V_{\text {harm }}=\frac{1}{2} r^{2}, V_{\text {anh }} & =\frac{1}{p} r^{p} \quad(p=3,4, \ldots)
\end{aligned}
$$

and the truncated Morse potential

$$
V(r)=\frac{1}{2} r^{2}-\frac{1}{2} r^{3}+\frac{7}{24} r^{4}
$$

which is the Taylor expansion of the Morse potential $\frac{1}{2}\left(e^{-r}-1\right)^{2}$. The last term in Eq. (11) represents the influence of external forces and is given by the expression

$$
U_{e x t}=-\sum_{n} \xi_{n}(t)\left(x_{n+1}-x_{n}\right)
$$

To take into account damping effects in soliton dynamics we introduce the dissipation function $\Psi$ 18]. In the case of the Stokes friction when the damping occurs due to interaction of the particles with a viscous environment(outer friction) the dissipation function depends on the velocity of the particles (Stokes law) and has the form

$$
\Psi_{s}=\frac{1}{2} \nu \sum_{n} \dot{x}_{n}^{2}(t)
$$


where $\nu$ is the damping constant. In the case of internal friction (we will call this type of friction hydrodynamical), which is due to irreversible processes taking place within the system, the dissipation function depends on the time derivatives of the relative displacements and is given by the expression

$$
\Psi_{h}=\frac{1}{2} \nu \sum_{n}\left(\dot{x}_{n+1}(t)-\dot{x}_{n}(t)\right)^{2} .
$$

The function (8) is the discrete version of the dissipation function which is usually used in macroscopic elasticity theory 18].

It is necessary to point out that if we consider the soliton as a wave packet, its dynamics in the presence of Stokes damping shows that the long-wave components of the spectrum do not propagate (App. A). Thus the soliton decomposes after a time in the order of $1 / \nu$. This feature does not show up with the hydrodynamical damping, if $\nu<1$ (see App. A).

The equations of motion for the lattice displacements $x_{n}(t)$ in the presence of damping have the form

$$
\frac{d}{d t} \frac{\partial L}{\partial \dot{x}_{n}}-\frac{\partial L}{\partial x_{n}}+\frac{\partial \Psi}{\partial \dot{x}_{n}}=0
$$

Substituting Eqs (1) - (8) into Eq. (91), the equations of motion for the relative displacement $u_{n}=x_{n+1}-x_{n}$ can be written as

$$
\begin{aligned}
& \ddot{u}_{n}=V^{\prime}\left(u_{n+1}\right)-2 V^{\prime}\left(u_{n}\right)+V^{\prime}\left(u_{n-1}\right)+ \\
& \xi_{n+1}(t)+\xi_{n-1}(t)-2 \xi_{n}(t)+D_{n}
\end{aligned}
$$

where $V^{\prime}(u)$ is the derivative of $V$ with respect to its argument $u$ and the damping term $D_{n}$ is determined by

$$
D_{n}= \begin{cases}-\nu \dot{u}_{n} & \text { for Stokes damping } \\ \nu\left(\dot{u}_{n+1}+\dot{u}_{n-1}-2 \dot{u}_{n}\right) & \text { for hydrodynamical } \\ \text { damping }\end{cases}
$$

In order to obtain a analytical solution of the nonlinear system of equations (10) we apply the quasi-continuum approximation proposed in [19] (see also [20, 21]). Regarding $n$ as a continuous variable $\left(n \rightarrow x, u_{n}(t) \rightarrow u(x, t)\right)$, Eq. (10) we obtain a damped and forced Boussinesq (Bq) equation (see App. B):

$$
\partial_{t}^{2} u-\partial_{x}^{2} u-\partial_{t}^{2} \partial_{x}^{2} u-\partial_{x}^{2}(f(u))=\nu_{m} \partial_{x}^{m} \partial_{t} u+\partial_{x}^{2} \xi(x, t)
$$


where $\partial_{x}$ and $\partial_{t}$ are the derivatives with respect to $x$ and $t$, respectively;

$$
f(u)=\frac{d V(u)}{d u}-u
$$

is a nonlinear force and the right-hand-side of Eq. (11) represents the damping in the system and the action of an external force. The case $m=0$ corresponds to the Stokes damping while the case $m=2$ corresponds to the hydrodynamical damping:

$$
\nu_{m}= \begin{cases}-\nu & \text { if } m=0 \\ \nu & \text { if } m=2 .\end{cases}
$$

\section{Multiple scale expansion}

We are interested in how the dynamics and the behavior of the soliton is affected by the two types of damping $(m=0,2)$. So we consider both the position of the soliton center of mass as a function of time, $X(t)$, and its shape for $t>0$. We make a travelling wave ansatz $u(x, t)=u(x-X(t))$ and use a multiple-scale perturbation expansion, developed in detail in App. $\mathbf{C}$, for a perturbed Bq equation

$$
\partial_{t}^{2} u-\partial_{x}^{2} u-\partial_{t}^{2} \partial_{x}^{2} u-\partial_{x}^{2}(f(u))=\epsilon F(x, t)
$$

where $\epsilon F(x, t)$ is the perturbation term with

$$
F(x, t)=\nu_{m} \partial_{x}^{m} \partial_{t} u+\partial_{x}^{2} \xi(x, t) .
$$

We seek an asymptotic solution of the form

$$
u=u_{0}+\epsilon u_{1}+\epsilon^{2} u_{2}+\cdots
$$

with

$$
c=c_{0}+\epsilon c_{1}+\epsilon^{2} c_{2}+\cdots
$$

where $c=-\partial_{t} X(t)$ is the velocity of the soliton. $\epsilon$ is a factor introduced for convenience in the analytical calculations. The case $\epsilon=0$ $\left(u=u_{0}\right)$ reduces Eq. (14) to the unperturbed Bq equation

$$
\partial_{t}^{2} u_{0}-\partial_{x}^{2} u_{0}-\partial_{t}^{2} \partial_{x}^{2} u_{0}-\partial_{x}^{2}\left(f\left(u_{0}\right)\right)=0
$$


which is the well-known improved Boussinesq (IBq) equation [19, 20, 22]. When $\epsilon=1$ we recover the damped and forced Bq equation (11). In order to interpret the multiple-scale perturbation results we must set $\epsilon=1$ and assume that the terms on the r.h.s of (11) are small enough. So, we must restrict ourselves to small values of the damping constant $\nu$.

In what follows we restrict our study to the damped Bq equation

$$
\partial_{t}^{2} u-\partial_{x}^{2} u-\partial_{t}^{2} \partial_{x}^{2} u-\partial_{x}^{2}(f(u))=\nu_{m} \partial_{x}^{m} \partial_{t} u
$$

The study of the effect of external forces, particularly stochastic forces, exceeds the frame of this paper and will be published later.

From the multiple-scale perturbation analysis we obtain that there are two compatibility conditions: One of them follows from the order $\epsilon^{1}$ of perturbation, Eq. (67); And the other one from the order $\epsilon^{2}$, Eq. (77). Both are valid for arbitrary potential $V(u)$.

Inserting the potential (4) or (5), together with the corresponding one soliton solution, into the compatibility conditions we get a set of two ordinary differential equations of motion (ODEs). These ODEs govern the time evolution of the order $\epsilon^{0}$ and $\epsilon^{1}$ of velocity perturbation, namely $c_{0}$ and $c_{1}$, respectively.

\subsection{The power-like anharmonic potential}

The expression (63) is the one-soliton solution of the IBq equation (18) with the power-like anharmonic potential (41). Substituting this solution in Eq. (67) and Eq. (77) yields

$$
\begin{gathered}
\dot{c}_{0}= \begin{cases}-\frac{(p-2) \nu c_{0}\left(c_{0}^{2}-1\right)}{6-3 p+2 p c_{0}^{2}} & m=0 \\
-\frac{(p-2)^{2} \nu\left(c_{0}^{2}-1\right)^{2}}{(p+2) c_{0}\left(6-3 p+2 p c_{0}^{2}\right)} & m=2\end{cases} \\
\dot{c}_{1}=-\nu \frac{(p-2)\left(3(p-2)+(18-7 p) c_{0}^{2}+2 p c_{0}^{4}\right)}{\left(6-3 p+2 p c_{0}^{2}\right)^{2}} c_{1} \\
+\quad \nu^{2} \frac{2(p-2) \sqrt{\pi} c_{0} \Gamma\left(\frac{p-1}{p-2}\right)^{2} \Gamma\left(\frac{2+p}{2 p-4}\right)}{\sqrt{c_{0}^{2}-1}\left(6-3 p+2 p c_{0}^{2}\right)^{4} \Gamma\left(\frac{p}{p-2}\right) \Gamma\left(\frac{p}{2 p-4}\right)^{2}} \\
\times \quad\left(6(p-2)^{3}-2(p-2)^{2}(-21+16 p) c_{0}^{2}\right.
\end{gathered}
$$




$$
\begin{aligned}
& +p\left(104-122 p+35 p^{2}\right) c_{0}{ }^{4}+p\left(16+14 p-13 p^{2}\right) c_{0}{ }^{6} \\
& \left.+2 p^{3} c_{0}{ }^{8}\right) \quad \text { for } m=0
\end{aligned}
$$

and

$$
\begin{aligned}
& \dot{c}_{1}=-\frac{\nu c_{1}(p-2)^{2}\left(c_{0}^{2}-1\right)}{(p+2)^{2} c_{0}^{2}\left(6-3 p+2 p c_{0}^{2}\right)^{2}}\left(-3\left(p^{2}-4\right)\right. \\
& \left.-\quad 3\left(-12-4 p+p^{2}\right) c_{0}^{2}+2 p(p+2) c_{0}^{4}\right) \\
& +\quad \nu^{2} \frac{2(p-2)^{2} \sqrt{\pi}\left(c_{0}^{2}-1\right)^{3 / 2} \Gamma\left(\frac{p-1}{p-2}\right)^{2} \Gamma\left(\frac{p+2}{2 p-4}\right)}{(2+p)^{2} c_{0}^{3}\left(6-3 p+2 p c_{0}^{2}\right)^{4} \Gamma\left(\frac{p}{p-2}\right) \Gamma\left(\frac{p}{2 p-4}\right)^{2}} \\
& \times \quad\left(3(p-2)^{4}-3(p-2)^{3}(-10+11 p) c_{0}^{2}\right. \\
& +\quad(p-2)^{2} p(-8+43 p) c_{0}^{4}+p\left(-32+84 p-17 p^{3}\right) c_{0}^{6} \\
& \left.+\quad 2 p^{3}(6+p) c_{0}^{8}\right) \quad \text { for } m=2 \\
& \hline
\end{aligned}
$$

where $\Gamma(\cdot)$ is the gamma function.

The set of ODEs (20-22) together with

$$
\dot{X}=c \quad \text { where } \quad c=c_{0}+c_{1}
$$

constitute the complete set of ODEs which determine $X(t)$ as a function of time.

\subsection{The truncated Morse potential}

The one-soliton solution of the IBq with the truncated Morse potential is

$$
u_{0}=\frac{A}{1+B \sinh ^{2}\left(\frac{\eta}{2}\left(x-c_{0} t\right)\right)}
$$

where

$$
A=\frac{ \pm 6\left(c_{0}^{2}-1\right)}{\mp 3+\sqrt{21 c_{0}^{2}-12}} \quad, \quad B=\frac{2 \sqrt{21 c_{0}^{2}-12}}{\mp 3+\sqrt{21 c_{0}^{2}-12}}
$$

and

$$
\eta=\frac{\sqrt{c_{0}^{2}-1}}{c_{0}}
$$


The upper sign means the soliton produces a rarefaction of the lattice, and the lower sign means compression. In this paper we consider only the compressional case. By inserting Eq. (24) in Eq. (67) and Eq. (77) we get a set of ODEs. They are rather cumbersome and therefore we make a further approximation by considering only the soliton dynamics close to the sound velocity. So we expand the ODEs in a Taylor series around the sound velocity where $O\left(c_{0}^{3}\right)$ terms are neglected. In the Stokes damping case the ODEs take the form

$$
\begin{aligned}
& \dot{c}_{0} \quad+\frac{68}{45} \nu c_{0}-\frac{19}{45} \nu c_{0}^{2}-\frac{49}{45} \nu=0 \\
& \dot{c}_{1} \quad-\frac{2 \nu\left(-101+41 c_{0}\right)}{15\left(5+7 c_{0}\right)} c_{1} \\
& -\frac{2 \nu^{2} \sqrt{2}\left(-39-1112 c_{0}+851 c_{0}^{2}\right)}{225 \sqrt{c_{0}-1}\left(5+7 c_{0}\right)}=0,
\end{aligned}
$$

and in the hydrodynamical damping case $(m=2)$

$$
\begin{aligned}
& \dot{c}_{0} \quad-\frac{8}{15} \nu c_{0}+\frac{4}{15} \nu c_{0}^{2}+\frac{4}{15} \nu=0 \\
& \dot{c}_{1} \quad+\frac{32 \nu\left(c_{0}-1\right)}{5\left(5+7 c_{0}\right)} c_{1}-\frac{176 \sqrt{2} \nu^{2}\left(c_{0}-1\right)^{\frac{3}{2}}}{75\left(5+7 c_{0}\right)}=0 .
\end{aligned}
$$

These approximations of the exact equations are good within a range of velocities $1<c_{0} \lesssim 1.1$

Notice that either Eqs. (26) or Eqs. (27), together with Eq. (23), constitute a complete set of equations of motion for $X(t)$.

\section{Numerical simulations}

In order to verify these theoretical results for both the power-like and the Morse potentials we have performed molecular dynamics simulation for the discrete monatomic chain which is governed by Eq. (10). Moreover we have performed simulations on the level of the quasicontinuum limit, namely with the damped Bq equation (19). For the damped Bq system we have used finite-difference discretization in the space-domain [23] (see App. D for details). The time integration in

both kinds of simulations was carried out by using the Heun method 
[24]. In order to start the simulations at $t=0$ we have used onesoliton solutions of the IBq equation. Since for some cases we need a long simulation time and the solitons are supersonic, we have used periodic boundary conditions to reduce the size of our system. In fact, we have used a chain with 1500 lattice points in the molecular dynamics simulation; and in the damped-Bq-simulation the length of the system has been $L=1000$ with $\Delta x=0.25$. We remark here that the solitons are bounded even when they develop a tail in the presence of perturbations, because the rear of this tail vanish. As the soliton including its tail is bounded in our finite system we are allowed to use periodic boundaries in our codes. The length of the tail grows with time, so we have considered not too long simulation times to avoid a possible overlapping between the rear of the tail and the front of the soliton. The other parameters have been $\Delta t=10^{-2}$ in the molecular dynamics simulation and $\Delta t=10^{-1}$ in the damped-Bq-simulation.

We have checked the accuracy of our codes by calculating the conserved quantity

$$
\int_{-\infty}^{\infty} u(x, t) d x
$$

which is valid not only for the free soliton case $\left(\nu_{m}=0\right)$ but also for the hydrodynamical damping case $(m=2)$. For the longest simulation time the variation of this conserved quantity has been lower than $4 \times 10^{-9} \%$ in the molecular dynamics simulation and lower than $2 \times 10^{-13 \%}$ in the damped-Bq-simulation. Notice that this conserved quantity can be calculated in a numerical window as long as there is not overlap between its tail and the front of the soliton.

The center of the soliton in both types of simulation has been found by finding the three points $x_{i-1}, x_{i}$ and $x_{i+1}$ where $u\left(x_{i}\right)$ is the absolute discrete maximum or minimum depending on whether the soliton is rarefactive or compressional. Afterwards a parabola has been fitted to the three coordinates, namely $\left\{x_{i}, u\left(x_{i}\right)\right\}$, and we have defined the vertex of this parabola as the soliton center of mass.

We have chosen $\nu_{0}=-\nu=-10^{-3}$ and $\nu_{2}=\nu=10^{-2}$. The reason for choosing different values is that the Stokes damping has a stronger effect than the hydrodynamical damping for the same value $\nu$. 


\subsection{Soliton dynamics in the presence of hydro- dynamical damping $(m=2)$}

In the case of the power-like anharmonic potential we have performed simulations in specific cases, namely for cubic as well as for quartic anharmonicity. We have also performed simulations in the case of the truncated Morse potential.

In the cubic case $(p=3)$ in the presence of hydrodynamical damping Eqs.20-22) reduce to

$$
\begin{aligned}
& \dot{c}_{0}+\frac{\nu\left(c_{0}^{2}-1\right)^{2}}{15\left(-c_{0}+2 c_{0}^{3}\right)}=0 \\
& \dot{c}_{1} \quad-\nu \frac{\eta^{2}\left(-4-3 \eta^{2}+2 \eta^{4}+\eta^{6}\right)}{15\left(1+\eta^{2}\right)^{3}} c_{1} \\
&-\nu^{2} \frac{\eta^{3}\left(22+40 \eta^{2}+18 \eta^{4}+\eta^{6}\right)}{225\left(1+\eta^{2}\right)^{3}}=0
\end{aligned}
$$

where $\eta$ has been defined in (25). In same way, for the quartic case $(p=4)$ Eqs. (20,22) reduce to

$$
\begin{aligned}
& \dot{c}_{0}+\frac{\nu\left(c_{0}^{2}-1\right)^{2}}{3\left(-3 c_{0}+4 c_{0}^{3}\right)}=0 \\
& \dot{c}_{1} \quad-\nu \frac{\eta^{3}\left(-4-3 \eta^{2}+3 \eta^{4}\right)}{3\left(1+3 \eta^{2}\right)^{2}} c_{1} \\
& \quad-\nu^{2} \frac{\pi^{2} \eta^{5}\left(9+29 \eta^{2}+39 \eta^{4}+3 \eta^{6}\right)}{36\left(1+3 \eta^{2}\right)^{4}}=0 .
\end{aligned}
$$

In the case of the truncated Morse potential, which contains a combination of cubic and quartic anharmonicities, we have already got the corresponding set of simplified Eqs. (27). So depending on the type of anharmonicity we have solved either Eqs. (28) or Eqs. (29), or Eqs. (27) together with Eq. (23). The result from those numerical solutions is what we call theory's prediction.

In Fig. 1 we show several examples of the soliton position as a function of time. These examples follow from the two kinds of simulations and from theory's prediction. In particular Fig. 1 1a and Fig. 

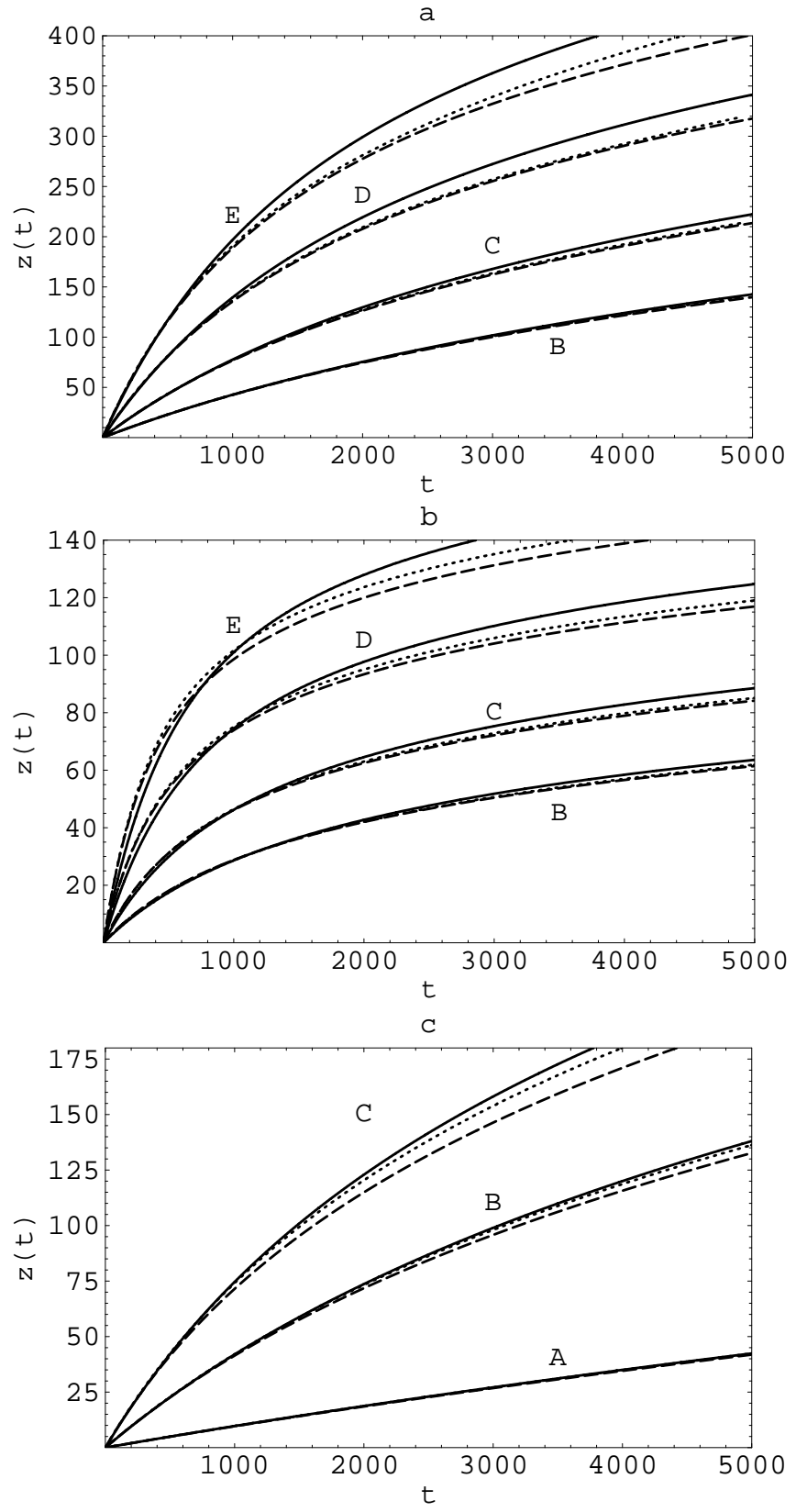

Figure 1: Soliton position in the sound velocity moving frame $z$ vs. time in the hydrodynamical damping case with $\nu=10^{-2}$. Solid line: molecular dynamics simulation, dashed line: theory's prediction (solution of Eqs.(2829)), dotted line: simulation for the Bq equation (19). Fig. (a) and (b) correspond to the cubic and quartic apharmonicities, respectively, and Fig. (c) corresponds to the truncated Morse potential. The uppercase letters A, $\mathrm{B}, \mathrm{C}, \mathrm{D}$, and $\mathrm{E}$ correspond to different initial velocities, namely $c(0)=1.01$, $1.05,1.1,1.2$ and 1.3 , respectively 
11b correspond to the soliton dynamics of the cubic and quartic cases, respectively. And Fig. Ic corresponds to the case of the truncated Morse potential. In each figure we show the soliton position for different initial velocities. These cases are denoted by uppercase letters. The position is plotted in the sound velocity moving frame, defined by $\mathrm{z}(t)=X(t)-t$. Notice that we consider lower initial velocities in the case of the truncated Morse potential (Fig. 1 $\mathrm{c}$ ), because in deriving Eqs. (27) further approximations have been made. In general for all the potentials we see that for low initial velocities (cases A, B and C) the lattice soliton position (solid-lines) is predicted rather well by theory's prediction (dashed lines) as well as by the position of the Bqsoliton (dotted lines). For higher initial velocities, namely $c(0)=1.2$ (cases D), the position of the lattice soliton agrees better with the position of the Bq soliton (dotted lines) than with the theory (dashed lines). And for even higher initial velocities, namely $c(0)=1.3$ (cases $\mathrm{E})$, quantitatively there is a clear difference between the three dynamics. However qualitatively they are similar. The reason for this behavior is that the quasi-continuum approximation naturally predicts better the dynamics of broad solitons than that of narrow ones [20]; and the solitons are narrower when the initial velocity is higher. Notice that in the cubic and quartic cases theory(dashed lines) predicts better the $\mathrm{Bq}$ soliton position (dotted lines) than the lattice soliton position (solid-lines). This is due to the fact that both the theory and the Bq equation have been derived in the framework of the quasicontinuum limit. This feature does not show up in the case of the truncated Morse potential due to the further approximations that we have made in the theory of this case.

\subsection{Soliton dynamics in the presence of Stokes damping $(m=0)$}

In this section we treat the same cases as in the previous section but with the soliton bearing systems in the presence of Stokes damping.

As in the previous section Eqs. (20 22) can be reduced depending on whether the potential has cubic or quartic anharmonicity. In particular for the cubic anharmonicity $(p=3)$

$$
\dot{c}_{0}+\frac{\nu c_{0}\left(c_{0}^{2}-1\right)}{6 c_{0}^{2}-3}=0
$$




$$
\dot{c}_{1} \quad+\nu \frac{\left(2+\eta^{2}+\eta^{6}\right)}{3\left(1+\eta^{2}\right)^{3}} c_{1}-\nu^{2} \frac{\left(-4+3 \eta^{2}+8 \eta^{4}+2 \eta^{6}\right)}{9 \eta\left(1+\eta^{2}\right)^{3}}=0 .
$$

In the same way the reduced set of equations for the quartic case is

$$
\begin{aligned}
\dot{c}_{0} \quad+\frac{\nu c_{0}\left(c_{0}^{2}-1\right)}{4 c_{0}^{2}-3}=0 \\
\dot{c}_{1}+\nu \frac{\left(\eta+3 \eta^{3}\right)^{2}\left(2-\eta^{2}+3 \eta^{4}\right)}{\eta^{2}\left(1+3 \eta^{2}\right)^{4}} c_{1}- \\
\nu^{2} \frac{\pi^{2}\left(-1-3 \eta^{2}-5 \eta^{4}+19 \eta^{6}+6 \eta^{8}\right)}{4 \eta\left(1+3 \eta^{2}\right)^{4}}=0
\end{aligned}
$$

And Eqs. (27) correspond to the case of the truncated Morse potential.

The theory's prediction of the soliton position follows from the numerical solution of either Eqs.(30) or Eqs.(31) or Eqs. (26) together with Eq. (23).

We show in Fig. 2 the same cases that we have treated in Fig. 1. We have also kept the same convention. In Fig. 2a we have not plotted the position of the $\mathrm{Bq}$ soliton because it agrees very well with the theoretical prediction (dashed line). In general we can extend the comments made for the hydrodynamical damping case to the present Stokes damping case. We only want to remark some relevant differences. First, in the present case we have plotted the soliton dynamics during the transient regime of the system, namely $t<1 / \nu$, in contrast to the hydrodynamical damping case where we have also considered times $t>>1 / \nu$. It is because the overdamp character of the Stokes damping, in fact, the lattice soliton is destroyed by the damping, namely for times $t \gtrsim 3 / \nu$. On the other hand, neither the $\mathrm{Bq}$ simulation nor the analytical results predict in a correct way the lattice soliton dynamics for times $t \gtrsim 1 / \nu$. And second, the agreement between the position of the lattice soliton (solid-line) and either the position of the Bq soliton (dotted line) or theory's prediction (dashed line) is not as good as in the hydrodymamical case. For instance, if we compare the results for the quartic case with $c_{0}(0)=1.2$ (Figs. 1]b and 2 $2 \mathrm{~b}$ : case D) we see that the agreement between simulations and theory is better for the hydrodynamical damping case than for the Stokes damping case. 

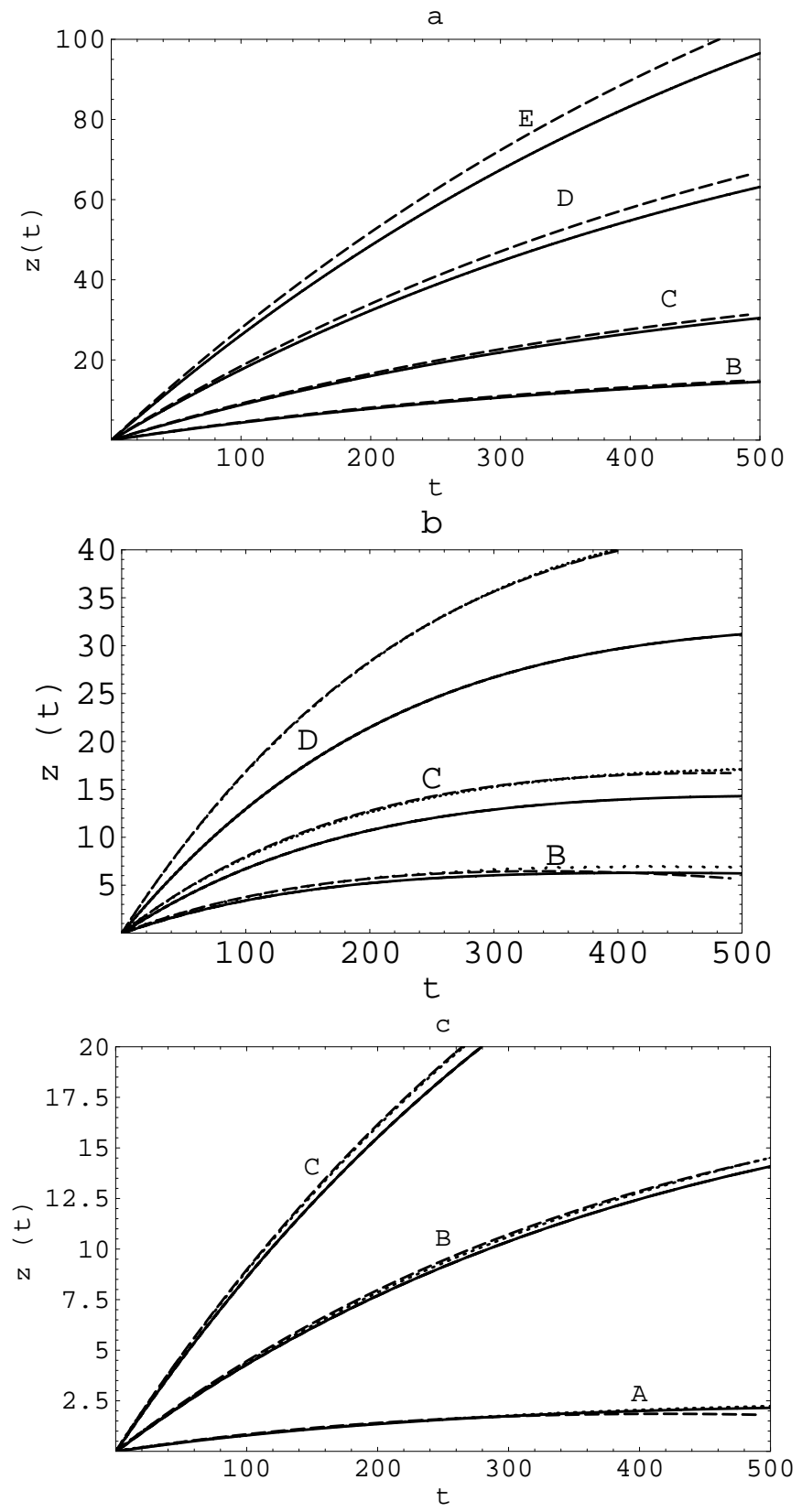

Figure 2: Soliton position in the sound velocity moving frame $z(t)$ vs. time in the Stokes damping case with $\nu=10^{-3}$. Solid line: molecular dynamics simulation, dashed line: theoretical prediction (solution of Eqs.(30-31)), dotted line: simulation of the Bq equation (19). Fig. (a) and Fig. (b) correspond to the cubic and quartic anhprmonicities respectively. And Fig. (c) corresponds to the truncated Morse potential. The uppercase letters A, B, $\mathrm{C}, \mathrm{D}$, and $\mathrm{E}$ correspond to different initial velocities, namely $c(0)=1.01$, $1.05,1.1,1.2$ and 1.3 , respectively 


\subsection{Soliton profile.}

Up to now we have analyzed the dynamics of solitons but not their shape under the influence of damping. The soliton profile for $t>0$ in the presence of either hydrodynamical damping or Stokes damping can be obtained by a multiple-scale perturbation theory (App. C). In first order the soliton solution reads

$$
u=u_{0}+u_{1}
$$

where $u_{0}$ is the unperturbed solution (63) and the function $u_{1}$ follows from Eqs. (79) and (84). As an example, the soliton solution in the case of a cubic potential with hydrodynamical damping reads

$$
u=u_{0}+\frac{1}{2} M+w+v
$$

with

$$
\begin{aligned}
w & =\operatorname{sech}^{2}(\phi)\left(A_{1}+A_{2} \phi \tanh (\phi)\right) \\
v & =A_{3} \phi \operatorname{sech}^{2}(\phi)+\left(A_{4}+\left(A_{5} \phi^{2}+A_{6}\right) \operatorname{sech}^{2}(\phi)\right) \tanh (\phi) \\
& +A_{7} \operatorname{Tanh}^{3}(\phi)
\end{aligned}
$$

where $\phi=\eta \theta / 2 . M$ and the coefficients $A_{i}, i=1, . ., 7$ depend only on the time and are written down explicitly in App. E. In order to have a look at this theoretical behavior compared with the results from the simulations we show in Fig. 3 two specific examples which belong to the cubic case. Figs. 3a and $3 \mathrm{~b}$ show snapshots of the soliton profile moving to the right side in the presence of hydrodynamical damping and Stokes damping, respectively. We see that in both examples the profiles are asymmetric and agree with each other rather well. The main feature which differs in both figures is that the amplitude of the tail which appears at the rear of the soliton in the presence of the hydrodynamical damping is positive, while in the other case it is negative. Notice that the theory's prediction of the Stokes damping case is not as good as in the hydrodynamical case where the dieviations are very small, in fact they are visible only in the center of the soliton.

\section{Discussion and conclusions}

In summary, in this work we have developed an analytical theory for the dynamics of lattice solitons on a monatomic chain under the influence of damping. We have considered Stokes and hydrodynamical 

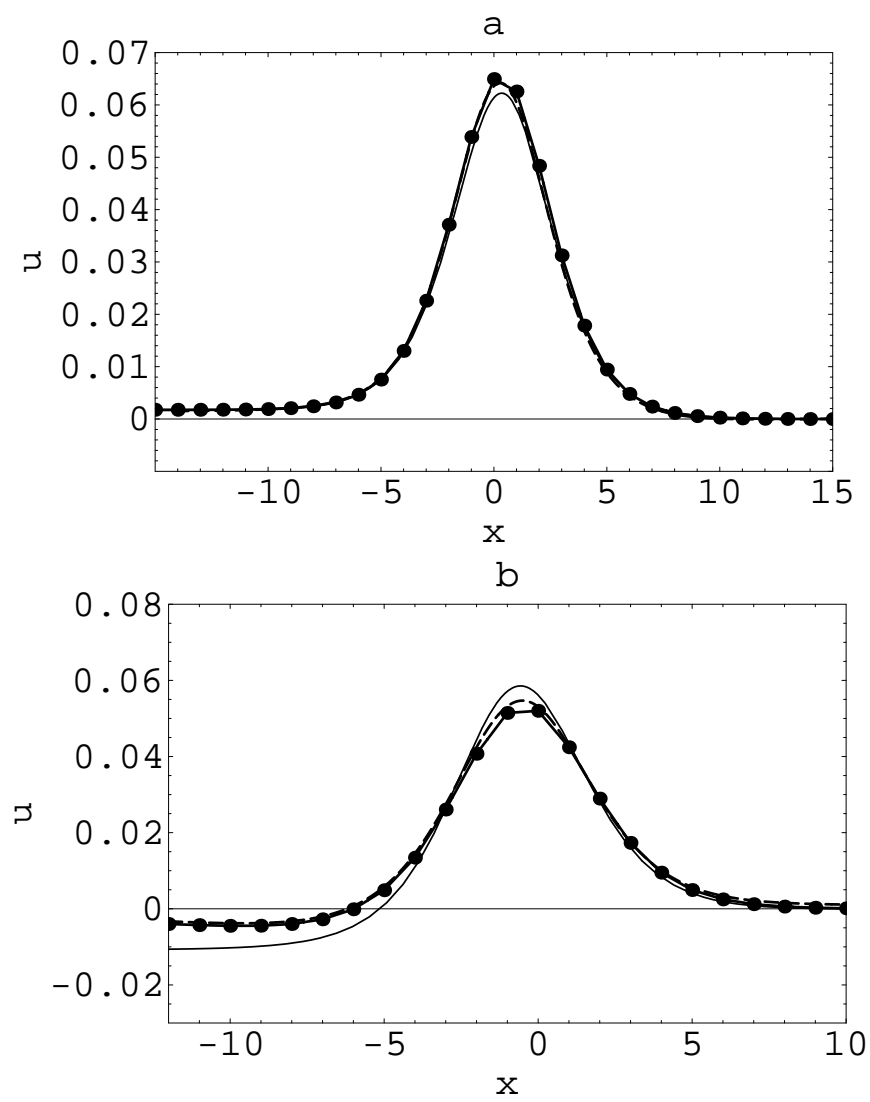

Figure 3: Snapshots of the soliton profile for the system with cubic anharmonicity. a: hydrodynamical damping case at $t=5000$ with $c(0)=1.05$, and $\nu=10^{-2}$. b: Stokes damping case at $t=500$ with $c(0)=1.1$ and $\nu=10^{-3}$. Solid circles: lattice soliton profile, dashed lines: Bq soliton profile, thin solid lines: theory's prediction. 
damping. In the quasi-continuum approximation the dynamics of the driven and damped anharmonic lattice has been described by a driven and damped Boussinesq equation. Our analytical approach has been based on the multiple-scale perturbation theory. We have derived sets of equations of motion corresponding to zero- and first order perturbations for the velocity. We have also calculated the first order perturbation for the soliton profile which develops a tail at the rear end. In order to check the validity of our results we have performed molecular dynamics simulation of the damped anharmonic lattice. We have also solved numerically the damped $\mathrm{Bq}$ equation. We have considered lattices with cubic and quartic anharmonicities. The soliton position has been defined as the position of the maximum of the soliton. We have observed that our theory predicts in a correct way the dynamics of lattice solitons when they propagate in a medium with either hydrodynamical or Stokes damping. This good agreement also holds for the soliton profiles. However, in the Stokes damping case our analysis is only done for the transient time, namely $t<1 / \nu$ where $\nu$ is the damping constant, because the soliton decomposes for larger times.

We have noticed that the quasi-continuum approximation describes in a better way the dynamics of the lattice solitons in the presence of hydrodynamical damping than in the case of the Stokes damping. This difference is due to the fact that in the hydrodynamical damping case the long-wave linear modes, which mostly contribute to the soliton dynamics, are underdamped (see Eq. (43)) while in the other case they are overdamped.

In general, the agreement between our theory and molecular dynamics simulation is mostly due to fact that our theory has been derived in the framework of the quasi-continuum limit. Moreover, this approach is better than earlier approximations made for the Kortewegde Vries equation [16], because higher soliton velocities can be considered.

\section{Acknowledgements}

We acknowledge support from DLR grant Nr.: UKR-002-99. Yu. Gaididei is grateful for the hospitality of the University of Bayreuth where this work was performed. 


\section{A Damping}

The Stokes damping does not permit the long wave components of a wave packet to propagate, while the hydrodynamical damping under a certain condition does not show this feature. This can be seen by means of a simple example: let us consider a harmonic monatomic chain with $2 N$ lattice points whose equations of motion for the relative displacements $u_{n}(t)$ in the presence of Stokes damping have the form

$$
\ddot{u}_{n}(t)=u_{n+1}(t)-2 u_{n}(t)+u_{n-1}(t)-\nu \dot{u}_{n}(t)
$$

with $n=1,2,3, \ldots, 2 N-1$. A travelling wave packet may be written as

$$
u_{n}(t)=\sum_{k=0}^{2 N-1} \tilde{u}_{k} e^{-i\left(\beta_{k} n-\omega_{k} t\right)}
$$

where $\beta_{k}$ is the wave number.

By inserting (35) in (34) we get $2 N$ equations of motion in $k$-space:

$$
\omega_{k}^{2}-i \nu \omega_{k}-\tilde{\gamma}_{k}=0
$$

where

$$
\tilde{\gamma}_{k}=2\left(1-\cos \left(\beta_{k}\right)\right)
$$

is a $k$-dependent function which satisfies

$$
0 \leq \tilde{\gamma}_{k} \leq 4
$$

Solving (36) we obtain

$$
i \omega_{k}=-\frac{\nu}{2} \pm i \sqrt{\tilde{\gamma}_{k}-\left(\frac{\nu}{2}\right)^{2}}
$$

Notice that for every finite value of $\nu>0$ there are small values of $k$ for which the condition of oscillation

$$
\tilde{\gamma}_{k}-\left(\frac{\nu}{2}\right)^{2}>0
$$

is not satisfied. In other words, parts of the wave packet do not propagate. 
However, this is not the case for the hydrodynamical damping. Here the equations of motion are

$$
\begin{aligned}
\ddot{u}_{n}(t)= & u_{n+1}(t)-2 u_{n}(t)+u_{n-1}(t) \\
& +\nu\left(\dot{u}_{n+1}(t)-2 \dot{u}_{n}(t)+\dot{u}_{n-1}(t)\right)
\end{aligned}
$$

with $n=1,2,3, \ldots, 2 N-1$ By using the ansatz (35) this set of equations reads in $k$-space

$$
\omega_{k}^{2}-i \nu \tilde{\gamma}_{k} \omega_{k}-\tilde{\gamma}_{k}=0
$$

with $k=1,2,3, \ldots, 2 N-1$.

$$
i \omega_{k}=-\frac{\nu}{2} \tilde{\gamma}_{k} \pm i \sqrt{\tilde{\gamma}_{k}\left(1-\left(\frac{\nu}{2}\right)^{2} \tilde{\gamma}_{k}\right)} .
$$

Here the condition of oscillation

$$
1-\left(\frac{\nu}{2}\right)^{2} \tilde{\gamma}_{k}>0
$$

is always satisfied, if $\nu<1$.

\section{B Quasi-continuum approximation}

The equations of motion of the lattice in the presence of dissipation and external forces are given by Eqs. (10). In order to compact the calculations we consider here a dissipation term $D_{n}$ containing both the Stokes and the hydrodynamical damping. So in this case the equations of motion read

$$
\begin{aligned}
& \ddot{u}_{n}=V^{\prime}\left(u_{n+1}\right)-2 V^{\prime}\left(u_{n}\right)+V^{\prime}\left(u_{n-1}\right)+ \\
& \xi_{n+1}(t)+\xi_{n-1}(t)-2 \xi_{n}(t)+\nu_{0} \dot{u}_{n}+ \\
& \nu_{2}\left(\dot{u}_{n+1}-2 \dot{u}_{n}+\dot{u}_{n-1}\right)
\end{aligned}
$$

where $\nu_{m}$, defined by Eq. (13), with $m=0,2$ are the damping constants of Stokes and hydrodynamical damping, respectively. This equation can be rewritten as

$$
\ddot{u}_{n}=\hat{\gamma}(n)\left(V^{\prime}\left(u_{n}\right)+\xi_{n}(t)+\nu_{2} \dot{u}_{n}\right)+\nu_{0} \dot{u}_{n}
$$


where

$$
\hat{\gamma}(n)=4 \sinh ^{2}\left(\frac{\partial_{n}}{2}\right)=4 \sinh ^{2}\left(\frac{a}{2} \partial_{x}\right)
$$

is a differential operator where $x=n a$ and $a$ is the lattice constant. At this point $x$ is regarded as a continuous variable, so $u_{n}(t) \rightarrow u(x, t)$ and $\xi_{n}(t) \rightarrow \xi(x, t)$. Taking into account that the function $4 \sinh ^{2}(d / 2) / d^{2}$ is smooth at $d=0$, we can multiply both sides of (46) by the operator $a^{2} \partial_{x}^{2} / 4 \sinh ^{2}\left(a \partial_{x} / 2\right)$, and expanding this operator as well as the operator on the r.h.s. in a Taylor series we get

$$
\begin{aligned}
& \partial_{t}^{2} u(x, t)=a^{2} \partial_{x}^{2} V^{\prime}+a^{2} \partial_{x}^{2} \xi(x, t)+a^{2} \lambda \partial_{x}^{2} \partial_{t}^{2} u(x, t) \\
& +\nu_{0} \partial_{t} u(x, t)-\nu_{0} a^{2} \lambda \partial_{x}^{2} \partial_{t} u(x, t)+\nu_{2} a^{2} \partial_{x}^{2} \partial_{t} u(x, t) .
\end{aligned}
$$

Setting $a=1$, scaling $x \rightarrow \sqrt{\lambda} x, t \rightarrow \sqrt{\lambda} t, \nu_{0} \rightarrow \nu_{0} / \sqrt{\lambda}, \nu_{2} \rightarrow \sqrt{\lambda} \nu_{2}$ and using the definition (12) we get

$$
\begin{aligned}
& \partial_{t}^{2} u(x, t)-\partial_{x}^{2} u(x, t)-\partial_{x}^{2} f(u(x, t))-\partial_{x}^{2} \partial_{t}^{2} u(x, t)= \\
& +\partial_{x}^{2} \xi(x, t)+\nu_{0} \partial_{t} u(x, t)-\nu_{0} \partial_{x}^{2} \partial_{t} u(x, t) \\
& +\nu_{2} \partial_{x}^{2} \partial_{t} u(x, t) .
\end{aligned}
$$

One of the Stokes damping terms can be neglected because the field $u(x, t)$ is slowly varying in space, therefore

$$
\left|\nu_{0} \partial_{t} u(x, t)\right|>>\left|\nu_{0} \partial_{x}^{2} \partial_{t} u(x, t)\right| .
$$

The estimate (50) has been confirmed by the numerical solution of Eq.(48) with and without the term $\nu_{0} \partial_{x}^{2} \partial_{t} u(x, t)$. In the rest of paper we consider separately either the Stokes damping case or the hydrodynamical case, therefore Eq. (49) can be written as

$$
\partial_{t}^{2} u-\partial_{x}^{2} u-\partial_{t}^{2} \partial_{x}^{2} u-\partial_{x}^{2}(f(u))=\nu_{m} \partial_{x}^{m} \partial_{t} u+\partial_{x}^{2} \xi(x, t)
$$

with $m=0,2$.

\section{Multiple-scale perturbation expan- sion}

In this appendix we develop a multiple-scale perturbation approach to the generalized Boussinesq-Burgers equation

$$
\partial_{t}^{2} u-\partial_{x}^{2} u-\partial_{t}^{2} \partial_{x}^{2} u-\partial_{x}^{2}(f(u))=
$$




$$
\epsilon\left(\nu_{m} \partial_{x}^{m} \partial_{t} u+\partial_{x}^{2} \xi(x, t)\right)
$$

where $f(u)=\frac{d V(u)}{d u}-u$ is a nonlinear force and the right-hand-side of this equation represents the damping in the system and the action of an external force. We consider two types of damping

$$
\nu_{m}= \begin{cases}-\nu & \text { if } m=0 \\ \nu & \text { if } m=2\end{cases}
$$

and $\epsilon$ is a small parameter. In our derivation we will follow the procedure which was proposed in [16] for the perturbed Korteweg-de-Vries equation.

By using the transformation to the moving frame of reference

$$
\begin{gathered}
\theta=x-X(T), \quad T=\epsilon t, \\
X(T)=\frac{1}{\epsilon} \int_{0}^{T} c\left(T^{\prime}\right) d T^{\prime}
\end{gathered}
$$

where $X(T)$ is the center of mass position of the soliton and $c(T)$ is its velocity which depend on the "slow" time variable $T$, Eq. (51) can be written in the form

$$
\begin{aligned}
& \left(c^{2} \partial_{\theta}^{2}-2 \epsilon c \partial_{\theta} \partial_{T}-\epsilon \dot{c} \partial_{\theta}+\epsilon^{2} \partial_{T}^{2}\right)\left(1-\partial_{\theta}^{2}\right) u \\
& -\partial_{\theta}^{2} u-\partial_{\theta}^{2}(f(u))=\epsilon\left(\nu_{m} \partial_{\theta}^{m}\left(\epsilon \partial_{T}-c \partial_{\theta}\right) u+\partial_{\theta}^{2} \zeta(\theta, T)\right)
\end{aligned}
$$

where $\cdot \equiv \frac{d}{d T}$ and the notation $\xi(x, t)=\zeta(\theta, T)$ was used. We seek an asymptotic solution of the form

$$
u=u_{0}+\epsilon u_{1}+\epsilon^{2} u_{2}+\cdots
$$

with

$$
c=c_{0}+\epsilon c_{1}+\epsilon^{2} c_{2}+\cdots
$$

Inserting Eqs (54) and (55) into Eq. (53) and collecting powers of $\epsilon$ we get

$$
\epsilon^{0} \text { : }
$$$$
\partial_{\theta}^{2}\left(\left(c_{0}^{2}-1\right) u_{0}-c_{0}^{2} \partial_{\theta}^{2} u_{0}-f\left(u_{0}\right)\right)=0,
$$ 
$\epsilon^{1}$ :

$$
\partial_{\theta}^{2}\left(\left(c_{0}^{2}-1\right) u_{1}-c_{0}^{2} \partial_{\theta}^{2} u_{1}-f^{\prime}\left(u_{0}\right) u_{1}\right)=\partial_{\theta} F_{1}
$$

$$
\begin{aligned}
F_{1} & =-\left(1-\partial_{\theta}^{2}\right)\left(2 c_{0} c_{1} \partial_{\theta}-2 c_{0} \partial_{T}-\dot{c}_{0}\right) u_{0} \\
& -\nu_{m} c_{0} \partial_{\theta}^{m} u_{0}+\partial_{\theta} \zeta(\theta, T)
\end{aligned}
$$

$\epsilon^{2}$ :

$$
\begin{gathered}
\partial_{\theta}^{2}\left(\left(c_{0}^{2}-1\right) u_{2}-c_{0}^{2} \partial_{\theta}^{2} u_{2}-f^{\prime}\left(u_{0}\right) u_{2}\right)=\partial_{\theta} F_{2} \\
-\quad\left(1-\partial_{\theta}^{2}\right) \partial_{T}^{2} u_{0}+\nu_{m} \partial_{\theta}^{m}\left(\partial_{T} u_{0}-c_{0} \partial_{\theta} u_{1}-c_{1} \partial_{\theta} u_{0}\right),
\end{gathered}
$$

$$
\begin{aligned}
F_{2}= & -\left(1-\partial_{\theta}^{2}\right)\left\{2 c_{0} c_{1} \partial_{\theta} u_{1}+\left(c_{1}^{2}+2 c_{0} c_{2}\right) \partial_{\theta} u_{0}-\right. \\
& \left.2 c_{0} \partial_{T} u_{1}-2 c_{1} \partial_{T} u_{0}-\dot{c}_{0} u_{1}-\dot{c}_{1} u_{0}\right\} \\
& +\frac{1}{2} \partial_{\theta}\left(f^{\prime \prime}\left(u_{0}\right) u_{1}^{2}\right) .
\end{aligned}
$$

Integrating twice Eq. (56) under vanishing boundary conditions for $u_{0}$ at infinity, we obtain the equation

$$
\left(c_{0}^{2}-1\right) u_{0}-c_{0}^{2} \partial_{\theta}^{2} u_{0}-f\left(u_{0}\right)=0 .
$$

In the case of the power-like anharmonic potential

$$
U_{a n h}=\frac{1}{p} u^{p}, \quad p>2
$$

the solution of Eq. (61) has the form

$$
u_{0}=\left(\frac{p}{2}\left(c_{0}^{2}-1\right)\right)^{1 /(p-2)} \operatorname{sech}^{2 /(p-2)}\left(\frac{p-2}{2 \ell} \theta\right)
$$

where the parameter $\ell=\frac{c_{0}}{\sqrt{c_{0}^{2}-1}}$ characterizes the width of the excitation.

Integrating Eq. (57) under vanishing boundary conditions for $\partial_{\theta} u_{1}$ at $\theta \rightarrow \pm \infty$ and for $u_{1} \rightarrow 0$ as $\theta \rightarrow \infty$ we get

$$
\left(c_{0}^{2}-1\right) u_{1}-c_{0}^{2} \partial_{\theta}^{2} u_{1}-f^{\prime}\left(u_{0}\right) u_{1}=-\int_{\theta}^{\infty} F_{1}(\bar{\theta}) d \bar{\theta} .
$$


The homogeneous part of Eq. (64) has two linearly independent solutions

$$
v_{1}=\partial_{\theta} u_{0}, \quad v_{2}=v_{1} \int_{0}^{\theta} \frac{d \bar{\theta}}{\left(\partial_{\bar{\theta}} u_{0}\right)^{2}}
$$

with the Wronskian $v_{2} \partial_{\theta} v_{1}-v_{1} \partial_{\theta} v_{2}=1$. Taking into account that of these solutions only $v_{1}$ vanishes as $\theta \rightarrow \pm \infty$, the compatibility condition for a bounded solution of the inhomogeneous equation (64) is

$$
\int_{-\infty}^{\infty} d \theta v_{1}(\theta) \int_{\theta}^{\infty} F_{1}(\bar{\theta}) d \bar{\theta} \equiv \int_{-\infty}^{\infty} u_{0} F_{1} d \theta=0
$$

Inserting Eq. (58) into Eq. (66) we obtain that the compatibility condition in the order $\epsilon^{1}$ has the form

$$
\partial_{T}\left(c_{0}\left\langle u_{0}^{2}+\left(\partial_{\theta} u_{0}\right)^{2}\right\rangle\right)=\nu_{m} c_{0}\left\langle u_{0} \partial_{\theta}^{m} u_{0}\right\rangle+\left\langle u_{0} \partial_{\theta} \zeta\right\rangle
$$

where the notation

$$
\langle g\rangle \equiv \int_{-\infty}^{\infty} g(\theta) d \theta
$$

was introduced. In the same way, we obtain from Eq. (59) that the compatibility condition in the order $\epsilon^{2}$ has the form

$$
\begin{aligned}
& \partial_{T}\left[2 c_{0}\left\langle u_{1}\left(1-\partial_{\theta}^{2}\right) u_{0}\right\rangle+c_{1}\left\langle u_{0}^{2}+\left(\partial_{\theta} u_{0}\right)^{2}\right\rangle\right]+ \\
& \left\langle u_{0}(\theta) \int_{\theta}^{\infty} d \bar{\theta} \partial_{T}^{2} u_{0}(\bar{\theta})\right\rangle+\frac{1}{2}\left(c_{0}^{2}-1\right) M^{2}= \\
& \nu_{m}\left(2 c_{0}\left\langle u_{1} \partial_{\theta}^{m} u_{0}\right\rangle+c_{1}\left\langle u_{0} \partial_{\theta}^{m} u_{0}\right\rangle-\left\langle u_{0} \partial_{T} \partial_{\theta}^{m-1} u_{0}\right\rangle\right) .
\end{aligned}
$$

Here

$$
M=\lim _{\theta \rightarrow-\infty} u_{1}(\theta)
$$


and as seen from Eq. (57) this quantity is determined by the expression

$$
\begin{aligned}
M= & -\frac{1}{c_{0}^{2}-1}\left\langle F_{1}(\theta)\right\rangle \\
= & -\frac{1}{c_{0}^{2}-1}\left(2 c_{0} \partial_{T}\left\langle u_{0}\right\rangle+\dot{c}_{0}\left\langle u_{0}\right\rangle-\nu_{0} \delta_{m 0} c_{0}\left\langle u_{0}\right\rangle\right. \\
& +\zeta(\infty, T)-\zeta(-\infty, T))
\end{aligned}
$$

Note that in deriving the compatibility condition (69) the relation

$$
\frac{1}{2}\left\langle u_{0} \partial_{\theta}\left(u_{1}^{2} f^{\prime \prime}\left(u_{0}\right)\right)\right\rangle=-\left\langle u_{1} \partial_{\theta}\left(u_{1} f^{\prime}\left(u_{0}\right)\right)\right\rangle
$$

and Eqs (57)-(58) were used.

Further simplification of the compatibility condition (69) may be achieved by using the relation

$$
\left(c_{0}^{2}-1-f^{\prime}\left(u_{0}\right)-c_{0}^{2} \partial_{\theta}^{2}\right) \frac{\partial u_{0}}{\partial c_{0}^{2}}=-\left(1-\partial_{\theta}^{2}\right) u_{0}
$$

which can be obtained by differentiating Eq. (61) with respect to $c_{0}^{2}$, and the relation

$$
\left(1-\frac{1}{c_{0}^{2}-1} f^{\prime}\left(u_{0}\right)-\ell^{2} \partial_{\theta}^{2}\right) \frac{\partial u_{0}}{\partial \ell^{2}}=\partial_{\theta}^{2} u_{0}
$$

which can be obtained from the equation

$$
\left(\ell^{2} \partial_{\theta}^{2}-1\right) u_{0}+\frac{1}{c_{0}^{2}-1} f\left(u_{0}\right)=0
$$

by differentiating it with respect to $\ell^{2}$. Thus by using Eqs (64) and (72) we get

$$
\begin{gathered}
\left\langle u_{1}\left(1-\partial_{\theta}^{2}\right) u_{0}\right\rangle=\frac{1}{2} c_{1} \frac{\partial}{\partial c_{0}}\left\langle\left(u_{0}^{2}+\left(\partial_{\theta} u_{0}\right)^{2}\right)\right\rangle \\
-\frac{1}{4 c_{0}} M\left(c_{0}^{2}-1\right) \frac{\partial\left\langle u_{0}\right\rangle}{\partial c_{0}} \\
-\frac{1}{2 c_{0}}\left\langle\frac{\partial u_{0}}{\partial c_{0}}\left(\zeta(\theta, T)-\frac{1}{2}(\zeta(-\infty, T)+\zeta(\infty, T))\right)\right\rangle .
\end{gathered}
$$


In the same way by using Eqs (64) and (73) we get

$$
\begin{array}{r}
\left\langle u_{1} \partial_{\theta}^{2} u_{0}\right\rangle=\frac{c_{0}^{2}-1}{4 c_{0}^{2}} M\left\langle u_{0}\right\rangle-\frac{c_{1}}{2 c_{0}}\left\langle u_{0}^{2}-\left(\partial_{\theta} u_{0}\right)^{2}\right\rangle+ \\
\frac{1}{2 c_{0}^{2}}\left\langle\theta \partial_{\theta} u_{0}\left(\zeta(\theta, T)-\frac{1}{2}(\zeta(-\infty, T)+\zeta(\infty, T))\right)\right\rangle .
\end{array}
$$

Inserting Eqs (75) and (76) into Eq. (69) we obtain that the compatibility condition in the order $\epsilon^{2}$ takes the form

$$
\begin{aligned}
& \frac{\partial}{\partial T}\left\{c_{1} \frac{\partial}{\partial c_{0}}\left(c_{0}\left\langle u_{0}^{2}+\left(\partial_{\theta} u_{0}\right)^{2}\right\rangle\right)\right. \\
- & \left.\frac{1}{2}\left(\left(c_{0}^{2}-1\right) M-\dot{c}_{0}\left\langle u_{0}\right\rangle\right) \frac{\partial\left\langle u_{0}\right\rangle}{\partial c_{0}}\right\}+\frac{1}{2}\left(c_{0}^{2}-1\right) M^{2} \\
- & \frac{1}{2} \dot{c}_{0}^{2}\left(\frac{\partial\left\langle u_{0}\right\rangle}{\partial c_{0}}\right)^{2}-\dot{c}_{0} \frac{\partial^{2}}{\partial c_{0}^{2}}\left\langle u_{0}(\zeta(\theta, T)\right. \\
- & \left.\left.\frac{1}{2}(\zeta(-\infty, T)-\zeta(\infty, T))\right)\right\rangle=\nu_{m} D_{m}
\end{aligned}
$$

where the right-hand-side is determined by the expressions

$$
\begin{aligned}
& D_{0}=\frac{c_{0}^{2}-1}{2 c_{0}} M\left(\left\langle u_{0}\right\rangle-c_{0} \frac{\partial\left\langle u_{0}\right\rangle}{\partial c_{0}}\right)+c_{1}\left\langle\left(\partial_{\theta} u_{0}\right)^{2}\right\rangle \\
+ & c_{1} c_{0} \frac{\partial}{\partial c_{0}}\left(\left\langle u_{0}^{2}\right\rangle+\left\langle\left(\partial_{\theta} u_{0}\right)^{2}\right\rangle\right)+\frac{\dot{c}_{0}}{2}\left\langle u_{0}\right\rangle \frac{\partial\left\langle u_{0}\right\rangle}{\partial c_{0}} \\
+ & \left\langle( \frac { \theta } { c _ { 0 } } \partial _ { \theta } u _ { 0 } - \frac { \partial u _ { 0 } } { \partial c _ { 0 } } ) \left(\zeta(\theta, T)-\frac{1}{2}(\zeta(-\infty, T)\right.\right. \\
+ & \zeta(\infty, T)))\rangle, \\
& D_{2}=\frac{c_{0}^{2}-1}{2 c_{0}} M\left\langle u_{0}\right\rangle-c_{1}\left\langle u_{0}^{2}\right\rangle \\
+ & \frac{1}{c_{0}}\left\langle\theta \partial_{\theta} u_{0}\left(\zeta(\theta, T)-\frac{1}{2}(\zeta(-\infty, T)+\zeta(\infty, T))\right)\right\rangle .
\end{aligned}
$$

To find how the soliton profile changes in the presence of damping it is convenient to represent the function $u_{1}$ in the form (see [16)

$$
u_{1}=\frac{1}{2} M+w+v
$$


where $w \rightarrow 0$ as $\theta \rightarrow \pm \infty$ while $v \rightarrow \mp \frac{1}{2} M$ as $\theta \rightarrow \pm \infty$. The functions $w$ and $v$ satisfy the equations

$$
\begin{gathered}
c_{0}^{2} \partial_{\theta}^{2} w+\left(f^{\prime}\left(u_{0}\right)-c_{0}^{2}+1\right) w=G^{w}(\theta) \\
c_{0}^{2} \partial_{\theta}^{2} v+\left(f^{\prime}\left(u_{0}\right)-c_{0}^{2}+1\right) v=G^{v}(\theta)
\end{gathered}
$$

where

$$
\begin{gathered}
G^{w}(\theta)=2 c_{0} c_{1}\left(1-\partial_{\theta}^{2}\right) u_{0}-\frac{1}{2} M f^{\prime}\left(u_{0}\right) \\
G^{v}(\theta)=-\int_{0}^{\theta}\left\{\left(1-\partial_{\bar{\theta}}^{2}\right)\left(2 c_{0} \partial_{T}+\dot{c}_{0}\right)-\nu_{m} c_{0} \partial_{\bar{\theta}}^{m}\right\} u_{0}(\bar{\theta}) d \bar{\theta}
\end{gathered}
$$

Using the functions (65) it is straightforward to see that the solutions of Eqs (80) and (81) are determined by the expressions

$$
\begin{gathered}
w=\frac{1}{c_{0}^{2}} \int_{0}^{\theta}\left(v_{1}(\theta) v_{2}(\bar{\theta})-v_{2}(\theta) v_{1}(\bar{\theta})\right) G^{w}(\bar{\theta}) d \bar{\theta}, \\
v=\frac{1}{c_{0}^{2}} \int_{0}^{\theta}\left(v_{1}(\theta) v_{2}(\bar{\theta})-v_{2}(\theta) v_{1}(\bar{\theta})\right) G^{v}(\bar{\theta}) d \bar{\theta} .
\end{gathered}
$$

and $c_{1}$ to be obtained via Eq. (77).

\section{Discretization of the $\mathrm{Bq}$ equation}

The Bq equations reads

$$
\begin{array}{r}
\partial_{t}^{2} u(x, t)-\partial_{x}^{2} u(x, t)-\partial_{t}^{2} \partial_{x}^{2} u(x, t)-\partial_{x}^{2}(f(u(x, t)))= \\
F(x, t)
\end{array}
$$

where $F(x, t)$ are external forces and/or dissipation. By defining the variable $v(x, t)=\partial_{t} u(x, t)$ Eq. (85) can be reduced to two partial differential equations of first order in time, namely

$$
\begin{aligned}
\partial_{t} v(x, t)= & \partial_{x}^{2} u(x, t)+\partial_{x}^{2} \partial_{t} v(x, t)+\partial_{x}^{2}(f(u(x, t))) \\
& +F(x, t) \\
\partial_{t} u(x, t)= & v(x, t) .
\end{aligned}
$$


By using finite-difference discretization in the space-domain Eqs. (86) take the form

$$
\begin{aligned}
\dot{v}_{i}(t)= & \frac{u_{i+1}(t)-2 u_{i}(t)+u_{i-1}(t)}{\Delta x^{2}} \\
& +\frac{\dot{v}_{i+1}(t)-2 \dot{v}_{i}(t)+\dot{v}_{i-1}(t)}{\Delta x^{2}} \\
& +\frac{\left.f\left(u_{i+1}(t)\right)\right)-2 f\left(u_{i}(t)\right)+f\left(u_{i-1}(t)\right)}{\Delta x^{2}} \\
& +F_{i}(t), \\
\dot{u}_{i}(t)= & v_{i}(t)
\end{aligned}
$$

where $\equiv \frac{d}{d t}, u_{i}(t)=u\left(x_{i}, t\right), v_{i}(t)=v\left(x_{i}, t\right), f\left(u_{i}(t)\right)=u^{n}\left(x_{i}, t\right)$ and $F_{i}(t)=F\left(x_{i}, t\right)$ with $n=2,3 . x_{i}=i \Delta x$ where $\Delta x$ is the mesh size of the space variable and $i=1,2, \cdots, N$. The length of the system $L=N \Delta x$. In the numerical integration process we use periodic boundary conditions, namely $u_{0}(t)=u_{N}(t)$ and $u_{N+1}(t)=u_{1}(t)$. The same boundaries are used for the variables $v_{i}(t)$ and $F_{i}(t)$. If we rewrite Eqs. (87) so

$$
\begin{aligned}
& -\dot{v}_{i+1}(t)+\left(\Delta x^{2}+2\right) \dot{v}_{i}(t)-\dot{v}_{i-1}(t)= \\
& u_{i+1}(t)-2 u_{i}(t)+u_{i-1}(t)+ \\
& f\left(u_{i+1}(t)\right)-2 f\left(u_{i}(t)\right)+f\left(u_{i-1}(t)\right)+ \\
& \Delta x^{2} F_{i}(t), \\
& \dot{u}_{i}(t)=v_{i}(t),
\end{aligned}
$$

they can be regarded as a vectorial equations so

$$
\begin{aligned}
\hat{\mathbf{A}} \dot{\mathbf{v}} & =\mathbf{G} \\
\dot{\mathbf{u}} & =\mathbf{v}
\end{aligned}
$$

where $\dot{u}_{i}$ and $\dot{v}_{i}$ are elements of the vectors $\dot{\mathbf{u}}$ and $\dot{\mathbf{v}}$, respectively. The elements $G_{i}$ of the vector $\mathbf{G}$ are the r.h.s. of (88) and the square matrix

$$
\hat{\mathbf{A}}=\left(\begin{array}{ccccccccc}
\Delta & -1 & 0 & 0 & \cdots & \cdots & 0 & 0 & -1 \\
-1 & \Delta & -1 & 0 & \cdots & \cdots & 0 & 0 & 0 \\
& \cdots & \cdots & \cdots & \cdots & \cdots & \cdots & \cdots & \\
0 & \cdots & 0 & -1 & \Delta & -1 & 0 & \cdots & 0 \\
& \cdots & \cdots & \cdots & \cdots & \cdots & \cdots & \cdots & \\
0 & 0 & \cdots & \cdots & 0 & 0 & -1 & \Delta & -1 \\
-1 & 0 & 0 & 0 & \cdots & \cdots & 0 & -1 & \Delta
\end{array}\right)_{N \times N}
$$


with $\Delta=\Delta x^{2}+2$. Notice that this tridiagonal matrix is cyclic because we use periodic boundary conditions [25]. From (90) we can derive

$$
\begin{aligned}
\dot{\mathbf{v}} & =\hat{\mathbf{A}}^{-1} \mathbf{G}, \\
\dot{\mathbf{u}} & =\mathbf{v},
\end{aligned}
$$

therefore at this stage we can use a classical integrator as for example the Heun algorithm in order to perform the numerical integration in time.

\section{E Coefficients}

The soliton solution in the case of cubic anharmonicity and hydrodynamical damping reads

$$
u=u_{0}+\frac{1}{2} M+w+v
$$

with

$$
\begin{aligned}
w= & \operatorname{sech}^{2}(\phi)\left(A_{1}+A_{2} \phi \tanh (\phi)\right) \\
v= & A_{3} \phi \operatorname{sech}^{2}(\phi)+\left(A_{4}+\left(A_{5} \phi^{2}+A_{6}\right) \operatorname{sech}^{2}(\phi)\right) \tanh (\phi) \\
& +A_{7} \operatorname{Tanh}^{3}(\phi)
\end{aligned}
$$

where

$$
\begin{gathered}
A_{1}=\frac{3 \nu}{5} \frac{\left(3-c_{0}^{2}\right) \sqrt{c_{0}^{2}-1}}{2 c_{0}^{2}-1}+3 c_{1} c_{0} \\
A_{2}=-\frac{3 \nu}{5} \frac{\left(3-c_{0}^{2}\right) \sqrt{c_{0}^{2}-1}}{2 c_{0}^{2}-1}-3 \frac{c_{1}}{c_{0}} \\
A_{3}=\frac{2 \nu \sqrt{c_{o}^{2}-1}}{5\left(2 c_{0}^{2}-1\right)} \quad A_{4}=-\frac{3}{8}\left(5 c_{0}^{2}-3\right) A_{3} \\
A_{5}=-\frac{1}{2 c_{0}^{2}} A_{3} \\
A_{7}=-\frac{1}{8}\left(5 c_{0}^{2}-3\right) A_{3} \quad M=-\frac{1}{8}\left(17-15 c_{0}^{2}\right) A_{3}
\end{gathered}
$$

The velocities $c_{0}$ and $c_{1}$ can be determined by Eqs. (28). 


\section{References}

[*] Permanent address: Bogolyubov Institute for Theoretical Physics, 252143 Kiev, Ukraine.

[1] M. Toda, Theory of Nonlinear Lattices, Springer-Verlag, BerlinHeidelberg-New York, (1981).

[2] F. G. Mertens, H. Büttner, in "Solitons", eds. S. E. Trullinger, V. E. Zakharov, V. L. Pokrovskii, North Holland, Amsterdam (1986).

[3] Fluctuation Phenomena:Disorder and Nonlinearity, edited by A. R. Bishop, S. Jiménez, and L. Vázquez (World Scientific, Singapore, 1995).

[4] Nonlinear Klein-Gordon and Schrödinger Systems: Theory and Applications, edited by L. Vázquez, L. Streit, and V. M. PerezGarcia (World Scientific, Singapore, 1996).

[5] St. Pnevmatikos, N. Flytzanis, and M. Remoissenet, Phys. Rev. E 33,2308 (1986).

[6] S. Jiménez and V.V. Konotop, Phys. Rev. B 60, 6465 (1999).

[7] V. Muto, P.S. Lomdahl and P.L. Christiansen, Phys. Rev. A 42, 7452 (1990).

[8] F. Mokross and H. Büttner, J. Phys. C 16, 4539 (1983)

[9] E. A. Jackson and A. D. Mistriotis, J. Phys. C 1,1223 (1989).

[10] N. Nishiguchi, Y. Kawada, and T. Sakuma, J.Phys.:Condens. Matter 4, 10227 (1992).

[11] Y. Ohtsubo, N. Nishiguchi, and T. Sakuma, J.Phys.:Condens. Matter 6, 3013 (1994).

[12] G. P. Tsironis, A.R. Bishop, A.V. Savin, and A.V. Zolotaryuk, Phys. Rev. E 60, 6610 (1999).

[13] J. Hietarinta, T. Kuusela, and B. Malomed, J. Phys. A: Math. Gen. 28, 3015 (1995).

[14] K.Ø. Rasmussen, B. Malomed, A.R. Bishop, N. Grønbech-Jensen, Phys. Rev. E 58, 6695 (1998).

[15] V.I. Karpman and E.M. Maslov, Sov. Phys. JETP 46,281 (1977); ibid. 48, 252 (1978). 
[16] R. Grimshaw and H. Mitsudera, Stud. Appl. Math. 90, 75 (1993).

[17] E. Mann, J. Math. Phys. 38, 3772 (1997).

[18] L.D. Landau and E. M. Lifshitz, Theory of Elasticity (Pergamon, Oxford, 1986).

[19] P. Rosenau, Phys. Lett. A 118, 222 (1986).

[20] D. Hochstrasser, F. G. Mertens, H. Büttner, Physica D35 259 (1989).

[21] A. Neuper, Yu. Gaididei, N. Flytzanis and F.G. Mertens, Phys. Rev. Lett. 75, 2240 (1995)

[22] V.G. Makhankov, Phys.Rep. 35,1 (1978).

[23] I.L. Bogolubsky, Comp.Phys.Commun. 13, 149 (1977)

[24] Peter E. Kloeden, Eckhard Platen, Numerical solution of Stochastic Differential Equations (Springer-Verlag, 1992)

[25] W. H. Press, S. A. Teuklsky, W. T. Vetterling and B. P. Flannery, Numerical Recipes in Fortran 2-nd edition (Cambridge University press, 1994). 